\title{
Improvements in resist performance towards EUV HVM
}

\author{
Oktay Yildirim*a, Elizabeth Buitrago ${ }^{\mathrm{b}}$, Rik Hoefnagels ${ }^{\mathrm{a}}$, Marieke Meeuwissen ${ }^{\mathrm{a}}$, Sander Wuister ${ }^{\mathrm{a}}$, \\ Gijsbert Rispens ${ }^{\mathrm{a}}$, Anton van Oosten ${ }^{\mathrm{a}}$, Paul Derks ${ }^{\mathrm{a}}$, Jo Finders ${ }^{\mathrm{a}}$, Michaela Vockenhuber ${ }^{\mathrm{b}}$ and Yasin \\ Ekinci $^{\mathrm{b}}$ \\ aASML Netherlands B.V., De Run 6501, 5504 DR Veldhoven, The Netherlands; ${ }^{\text {a }}$ Laboratory for \\ Micro- and Nanotechnology, Paul Scherrer Institute, CH-5232 Villigen PSI, Switzerland
}

\begin{abstract}
Extreme ultraviolet (EUV) lithography with $13.5 \mathrm{~nm}$ wavelength is the main option for sub-10nm patterning in the semiconductor industry. We report improvements in resist performance towards EUV high volume manufacturing. A local CD uniformity (LCDU) model is introduced and validated with experimental contact hole $(\mathrm{CH})$ data. Resist performance is analyzed in terms of ultimate printing resolution (R), line width roughness (LWR), sensitivity (S), exposure latitude (EL) and depth of focus (DOF). Resist performance of dense lines at $13 \mathrm{~nm}$ half-pitch and beyond is shown by chemical amplified resist (CAR) and non-CAR (Inpria YA Series) on NXE scanner. Resolution down to $10 \mathrm{~nm}$ half pitch (hp) is shown by Inpria YA Series resist exposed on interference lithography at the Paul Sherrer Institute. Contact holes contrast and consequent LCDU improvement is achieved on a NXE:3400 scanner by decreasing the pupil fill ratio. State-of-the-art imaging meets $5 \mathrm{~nm}$ node requirements for CHs. A dynamic gas lock (DGL) membrane is introduced between projection optics box (POB) and wafer stage. The DGL membrane will suppress the negative impact of resist outgassing on the projection optics by $100 \%$, enabling a wider range of resist materials to be used. The validated LCDU model indicates that the imaging requirements of the $3 \mathrm{~nm}$ node can be met with single exposure using a high-NA EUV scanner. The current status, trends, and potential roadblocks for EUV resists are discussed. Our results mark the progress and the improvement points in EUV resist materials to support EUV ecosystem.
\end{abstract}

Keywords: LCDU, EUV resists, CAR, Non-CAR, photoresist, EUV lithography, sub10 nm, 5nm node, 3nm node

\section{INTRODUCTION}

Extreme ultra-violet (EUV) lithography is preparing for high-volume manufacturing being the preferred option for beyond $10 \mathrm{~nm}$ node, offering significant reduction in process complexity ${ }^{1}$. ASML's $5^{\text {th }}$ generation EUV 0.33 NA scanner system NXE:3400B provides a resolution down to $13 \mathrm{~nm}$ half-pitch and $125 \mathrm{wph}$ throughput with a new flexible illuminator, having a minimum pupil fill ratio of $20 \%$ without transmission loss, improved lens aberration, and an improved EUV Source. For the longer term a larger numerical aperture of $>0.5$ is being developed ${ }^{1,2}$.

Sensitivity (S), resolution (R) and line width roughness (LWR) are very critical parameters for the EUV photoresist. Due to the trade-off relation between resolution, line-edge roughness and sensitivity, the so-called RLS triangle, it has been challenging to have simultaneous improvement in these resist performance parameters ${ }^{3,4,5,6,7}$. However this tradeoff relation is for a given resist at a given aerial image contrast and in principle can be altered by improving the optical contrast (via scanner) and/or improvement by resist. Photoresists are complex structures that undergo chemical changes upon interaction with EUV light. Current standard platforms are called as chemically amplified resists (CARs), which are described in many studies in detail ${ }^{5,7,8}$. CARs are mainly composed of organic materials with low EUV absorption, such as an acrylate or styrene based bulk polymer with protected side groups, and several functional chemical components such as photo acid generators (PAG) or quenchers. Secondary electrons created upon EUV absorption react with PAGs to create acids ${ }^{9,10}$. Acids diffuse and catalyze deprotection reaction in the polymer which leads to a chemical contrast between exposed and unexposed regions to be exploited in development step to create patterns. Due to the electron blur and acid diffusion, the chemical reaction can take place a few nanometers away from the EUV absorption site $^{8,11}$. To have smaller feature sizes, blur is limited with several methods such as using quenchers, employing smaller diffusivity acids or lowering baking temperatures. However, decreased blur results in increased need in the number of acids to be created, leading to increased EUV dose requirement. Having low LWR while using high sensitivity resist (high sensitivity=low dose) is another challenge for EUV resists, due to photon shot noise effects ${ }^{5,6,8,12}$.

Extreme Ultraviolet (EUV) Lithography VIII, edited by Eric M. Panning, Kenneth A. Goldberg, Proc. of SPIE Vol. 10143, 101430Q · C 2017 SPIE · CCC code: 0277-786X/17/\$18 · doi: 10.1117/12.2257415 
Metal Oxide based SnOx containing non-CAR Inpria resists are presented as EUV resist platforms with high absorptivity up to $20 \mu^{-1}\left(\sim 4 \mathrm{x}\right.$ higher than CAR) with high resolution and high etch resistance ${ }^{13,14}$. These platforms have a relatively simple material composition, SnOx clusters surrounded by organic ligands, and different activation mechanism compared to CARs. Upon EUV exposure the SnOx clusters crosslink and forms the resist pattern, whereas unexposed areas are dissolved ${ }^{13,14}$.

It has been challenging to describe a simple model capturing the real resist input parameters and aerial image contribution in a combined way to describe the local critical dimension uniformity (LCDU). Absorptivity and blur are key items which effects resist performance and eventually the LCDU regardless of the resist platform.

Using state-of-the-art resists, exposure and metrology tools we show EUV resist status and potential. With $\mathrm{CH}$ data we show the improved LCDU due to increased aerial image contrast. We introduce a LCDU model combining resist related items and scanner related items to explain LCDU. The validated model is further used to predict LCDU in high-NA case.

\section{EXPERIMENTAL}

\section{EUV Exposures at PSI and ASML:}

EUV interference lithography (EUV-IL) exposures are performed at PSI by illuminating a mask ${ }^{15}$ with transmissiondiffraction gratings by a spatially coherent beam of EUV light (13.5 $\mathrm{nm}$ wavelength) to produce periodic images by the interference of two diffracted beams ${ }^{16,17}$. The aerial image generated by EUV-IL is a sinusoidal pattern with high contrast.

At ASML: NXE:3400B scanner with $13 \mathrm{~nm}$ "leaf-shape" dipole $\mathrm{Y}$ and $12 \mathrm{~nm}$ "leaf-shape" dipole X pupils with $20 \%$ and $13.1 \%$ pupil fill ratios respectively, are used for NXE exposures. Results for nominal (0) mask biases are reported.

\section{Metrology at ASML:}

To measure the $100 \mathrm{~mm}$ diameter wafers exposed at PSI Hitachi CG-4000 CDSEM is used. For the 300mm wafers exposed at ASML, CG-5000 Hitachi CDSEM is used. Settings for CG4000 are selected in order to have similar shrinkage effect with CG5000 on the resists. Images reported for dense lines are LWR images.

CG-4000 CD measurement settings: $500 \mathrm{~V}, 300 \mathrm{kx} 100 \mathrm{k}$ magnification and $512 \times 512$ pixels.

CG-5000 CD measurement: 500V, 150kx49k magnification and 1024x1024 pixels.

CG-4000 LWR measurement settings: 500V, 160kx160k magnification and 1024x1024 pixels.

CG-5000 LWR measurement settings: 500V, 80kx80k magnification and 2048x2048pixels.

\section{Processing Information}

The processing conditions are determined based on the input from resist suppliers. CAR resists conditions for dense lines: $25 \mathrm{~nm}$ film thickness (FT), $130^{\circ} \mathrm{C} \backslash 60$ s soft bake (SB), $110^{\circ} \mathrm{C} \backslash 60$ s post exposure bake (PEB), 30s development with standard positive tone developer and rinse with rinse material. (Inpria YA Series) resist conditions: 20nm FT, $100^{\circ} \mathrm{C} \backslash 120 \mathrm{~s} \mathrm{SB}, 90^{\circ} \mathrm{C}-150^{\circ} \mathrm{C} \backslash 60 \mathrm{~s}-120 \mathrm{~s}$ PEB, development with negative tone developer, with $150^{\circ} \mathrm{C}$ hard bake (HB).

\section{RESULTS\&DISCUSSIONS}

\subsection{EUV Resist Targets for Resolution, Roughness and Sensitivity}

Table 1 shows targets for resolution, LWR and dose for dense lines (DL) and for contact holes (CH) over time. Timing is given by scanner readiness for the given resolution. In connection with the increasing resolution over time, the line width roughness (LWR) for lines and local critical dimension uniformity (LCDU) for $\mathrm{CH}$ requirements are also getting tighter. For 2017 the resolution target for DLs is $13 \mathrm{~nm}$, and for CHs 20nm. 
Table 1. EUV Resist Targets.

\begin{tabular}{|c|c|c|c|c|c|c|}
\hline & \multicolumn{2}{|c|}{ Target } & 2013 & 2015 & 2017 & 2020 \\
\hline \multirow{3}{*}{ 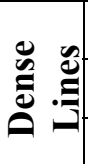 } & \multicolumn{2}{|c|}{ Resolution } & $22 \mathrm{~nm}$ & $16 \mathrm{~nm}$ & $13 \mathrm{~nm}$ & $8 \mathrm{~nm}$ \\
\hline & LWR & $<20 \%$ & $<4.4 \mathrm{~nm}$ & $<3.2 \mathrm{~nm}$ & $<2.6 \mathrm{~nm}$ & $<1.6 \mathrm{~nm}$ \\
\hline & Dose & $<20 \mathrm{~mJ} / \mathrm{cm}^{2}$ & $<20 \mathrm{~mJ} / \mathrm{cm}^{2}$ & $<20 \mathrm{~mJ} / \mathrm{cm}^{2}$ & $<20 \mathrm{~mJ} / \mathrm{cm}^{2}$ & $<20 \mathrm{~mJ} / \mathrm{cm}^{2}$ \\
\hline \multirow{3}{*}{ 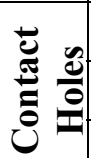 } & \multicolumn{2}{|c|}{ Resolution } & $24 \mathrm{~nm}$ & $24 \mathrm{~nm}$ & $20 \mathrm{~nm}$ & $18 \mathrm{~nm}$ \\
\hline & $\mathrm{LCDU}$ & $<15 \%$ & $<3.6 \mathrm{~nm}$ & $<3.6 \mathrm{~nm}$ & $<3.0 \mathrm{~nm}$ & $<2.7 \mathrm{~nm}$ \\
\hline & Dose & $<20 \mathrm{~mJ} / \mathrm{cm}^{2}$ & $<20 \mathrm{~mJ} / \mathrm{cm}^{2}$ & $<20 \mathrm{~mJ} / \mathrm{cm}^{2}$ & $<20 \mathrm{~mJ} / \mathrm{cm}^{2}$ & $<20 \mathrm{~mJ} / \mathrm{cm}^{2}$ \\
\hline
\end{tabular}

\subsection{LCDU and LCDU model}

Local variations, such as LCDU is becoming very critical especially at smaller dimensions and low dose values. In Fig.1 several factors affecting LCDU is shown. LCDU after lithography (ADI) has two major contributors, i.e. resist related items and aerial image contrast dependent on the lithography set-up. We developed a model mainly capturing these two major contributors. Resist blur and absorptivity are key parameters for all resist platforms, regardless of the type. PAG type/load is a CAR specific parameter and microscopic property such as core size is applicable to non-CAR resists containing nanoparticle or metal-oxo cluster cores. Post processing typically can improve LCDU significantly.

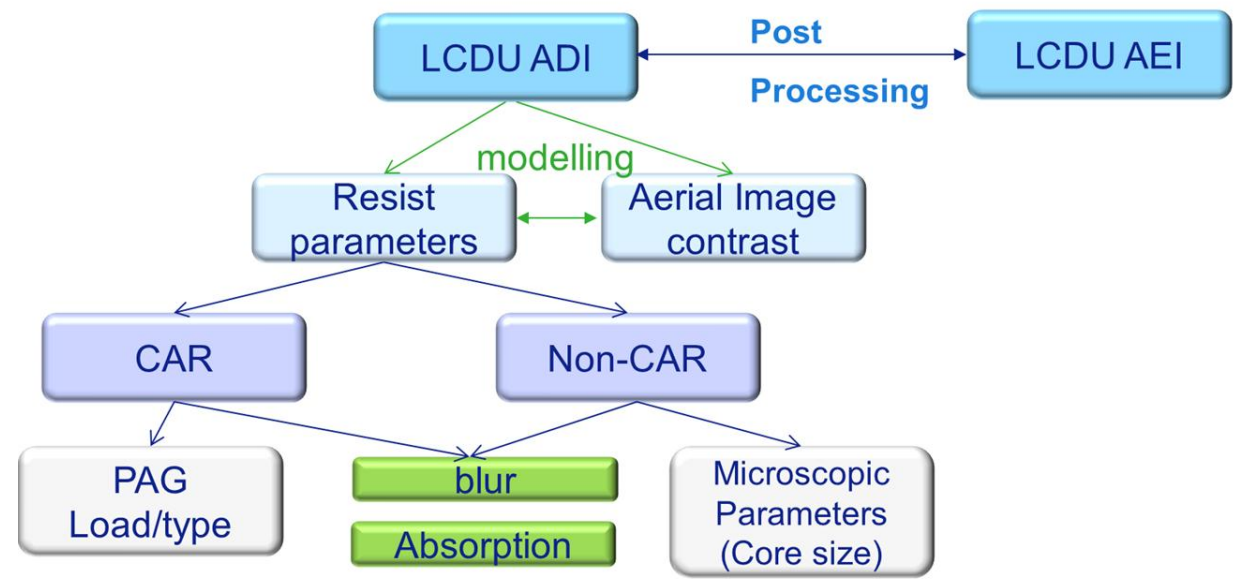

Blur and absorption are key parameters both for CAR and non-CAR resists

Figure 1. LCDU photon shot noise landscape.

Figure 2 highlights some underlying aspects of LCDU model. Dose variation is caused by stochastic variation of the number of absorbed photons. These dose fluctuations result in local CD variation. What matters regarding LCDU is the variation at the edge of the feature. Blur plays a key role to define the edge/the photon counting area. With more photon counting, less dose variation (left), thus lower LCDU is expected. On the other hand, blur results in loss of contrast (right). By properly defining the edge/photon counting area the model can be used to define LWR as well. The blur is the standard deviation of a Gaussian distribution $\sigma$. To count the absorbed photons a "top hat approximation" is used, where the probability is either 1 inside the effective radius and 0 outside. This radius, " $r$ " is $\sqrt{2}$ times bigger than the blur, " $\sigma$ ". 


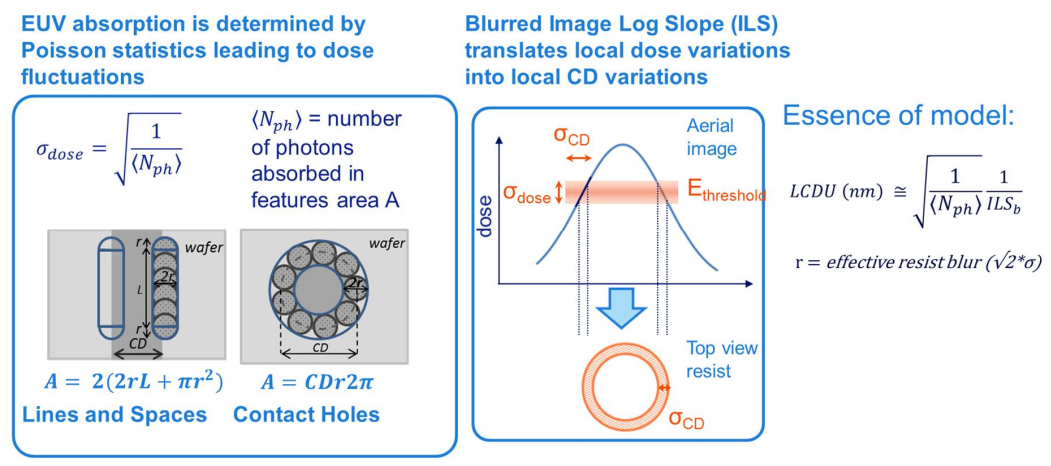

Figure 2. Explanation of photon shot noise model.

By changing the blur at LCDU model we observed that optimum blur is pitch dependent (Fig. 3). Increasing resist blur has two competing effects. First is loss of aerial image contrast leading to higher LCDU. Second, increase in the photon counting area leading to lower LCDU. The actual best possible LCDU value is determined by the trade-off relation between these two effects. The optimum blur is between (half-pitch)/5 and (half-pitch)/3.

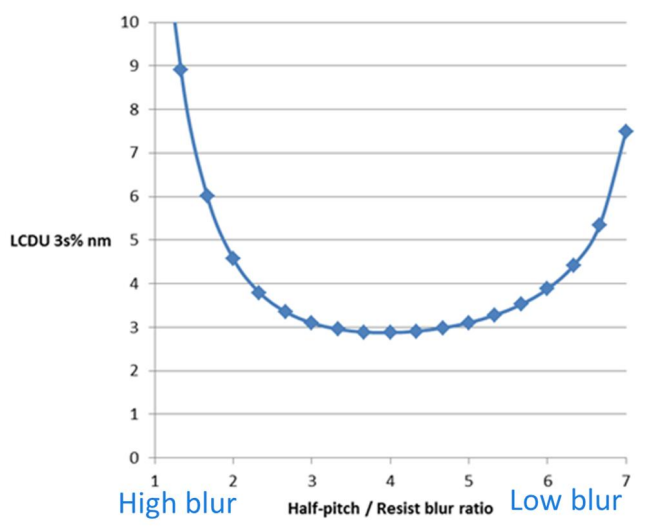

Figure 3. Optimum blur is pitch dependent. Graph created by using LCDU model.

Table 2. LCDU model parameters and resist assumptions.

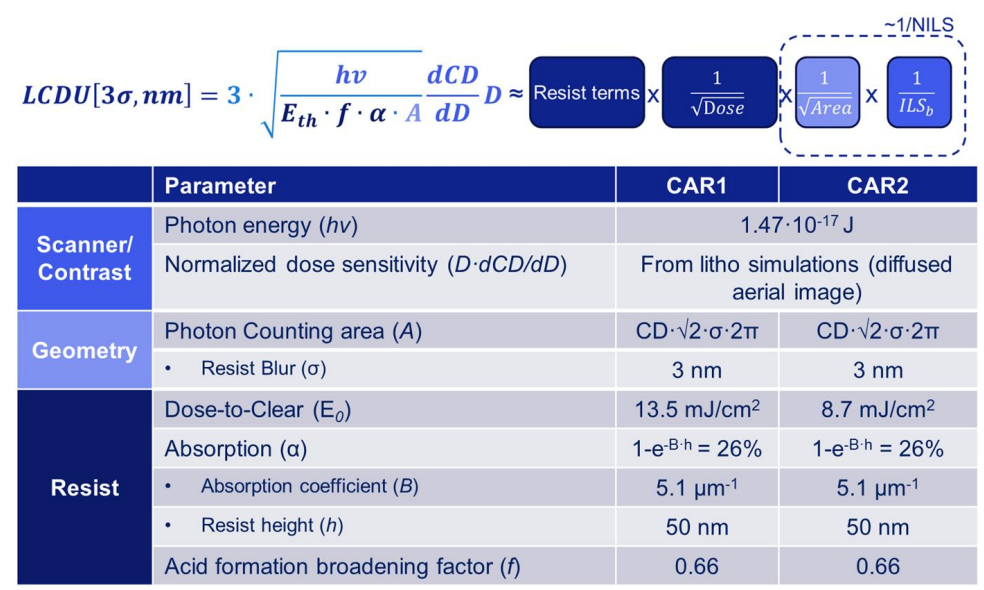

EO, DillB and thickness values are determined experimentally

Table 2 shows the parameters used in our LCDU model in more detail. In the model scanner related items and resist related items are combined to define LCDU. Normalized dose sensitivity (NDS) is calculated from lithography 
simulations. Blur defined (photon counting) area and NDS form the contrast part of the model. Resist specific parameters such as absorptivity (Dill $B$ ), $\mathrm{E}_{0}$ are also part of the model. Absorption is defined by resist thickness and Dill $B$. In the case shown in Table 2, $\mathrm{E}_{0}$ and Dill $B$ resist thickness are experimentally determined for two different resists.

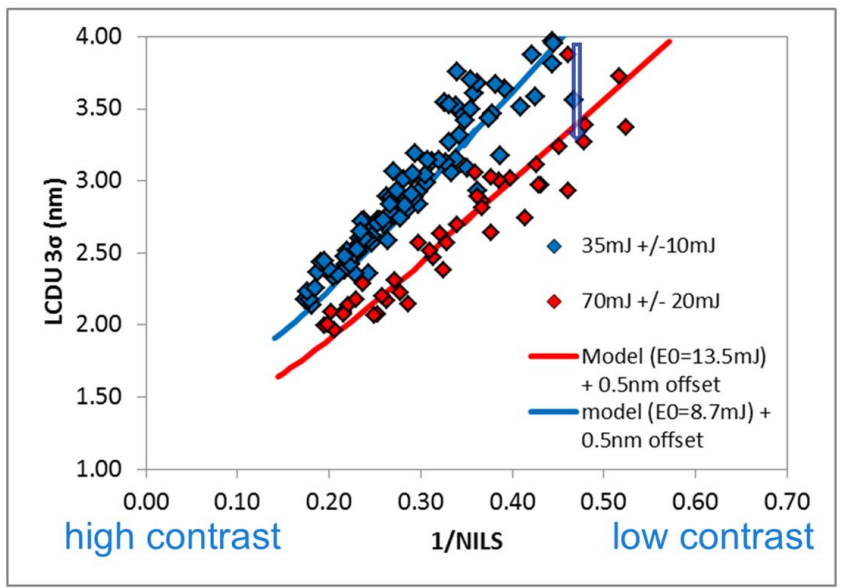

Figure 4. Experimental validation LCDU model. Local CDU in $\mathrm{nm} 3 \sigma$ for rectangular array of CH versus $1 /$ NILS (contrast). Two different resist formulations have been used. CHs of 22-35nm have been exposed on NXE:3300 using conventional illumination.

Measuring LCDU and plotting vs $1 /$ NILS $^{18}$ shows the strong dependency of LCDU on image contrast. As shown in Fig. 4, two different resists, which are explained in Table 2, both show same typical relation between LCDU and contrast: improving contrast lowers LCDU and/or dose. The solid lines passing through the data points are the model applied, using the real resist input parameters described in Table 2. We see a good match of LCDU model with the experimental data, validating that the model captures the slope very well. However, a $0.5 \mathrm{~nm}$ off set was needed to match the model to the data. At this stage we point out that the same $0.5 \mathrm{~nm}$ offset was used for the two different resists. This implies the strength of the model, in the sense that resist related items are captured by the model and having same offset hints a nonresist related source, e.g. metrology.

\subsection{Contrast Improvement}

Finders et al. ${ }^{18}$ showed that illumination mode can be adjusted to provide highest contrast. High aerial image contrast is essential to have high quality patterns, with lower roughness and lower dose. LWR and LCDU have multiple contributors such as mask, resist material, metrology and photon shot noise. The impact of photon shot noise depends strongly on the aerial image contrast. They used normalized image log-slope (NILS) as a measure of aerial image contrast. They showed that local critical dimension uniformity (LCDU) can be improved (which means smaller LCDU values) by increasing the contrast. Using smaller pupil fill ratio (PFR) illumination led to higher contrast and this resulted in smaller LWR (due to improved photon shot noise) and lower dose for $14 \mathrm{~nm}$ dense lines ${ }^{18}$.

Similarly, Kerkhof et al. ${ }^{19}$ used smaller pupilfill ratio illumination which enabled higher contrast. This led to lower LCDU values for CHs of 22 and $18 \mathrm{~nm} \mathrm{hp}$ as shown in Figure 5. On the top graph the simulations on illumination conditions of 22 and $18 \mathrm{~nm} \mathrm{CHs} \mathrm{are} \mathrm{shown.} \mathrm{Simulation} \mathrm{shows} \mathrm{that} \mathrm{with} \mathrm{the} \mathrm{reduced} \mathrm{PFR} \mathrm{increased} \mathrm{contrast} \mathrm{as} \mathrm{expected.}$ The experimental results in the below graph indeed shows the improved LCDU due to the contrast increase. Scanner improvement enables better imaging performance, in this case by improved aerial image contrast provided. 


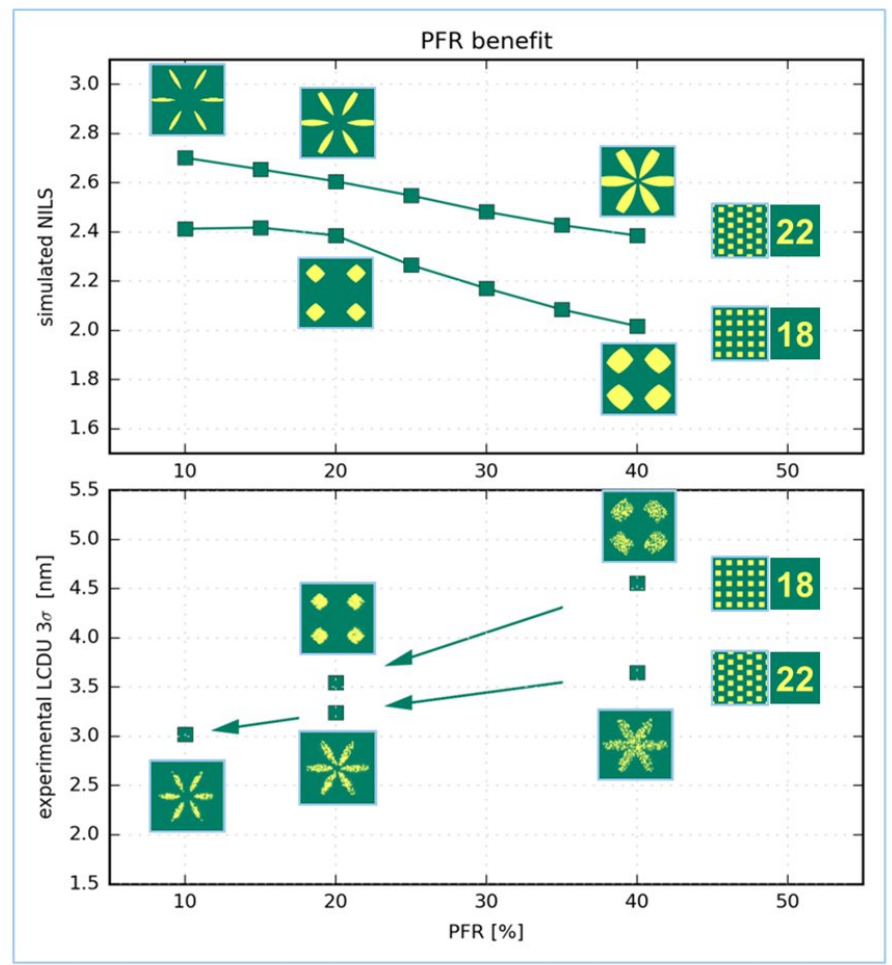

Figure 5. Simulation above: contrast is improved by reducing the pupil fill ratio for CHs. Experimental result below: high contrast leads to lower LCDU.

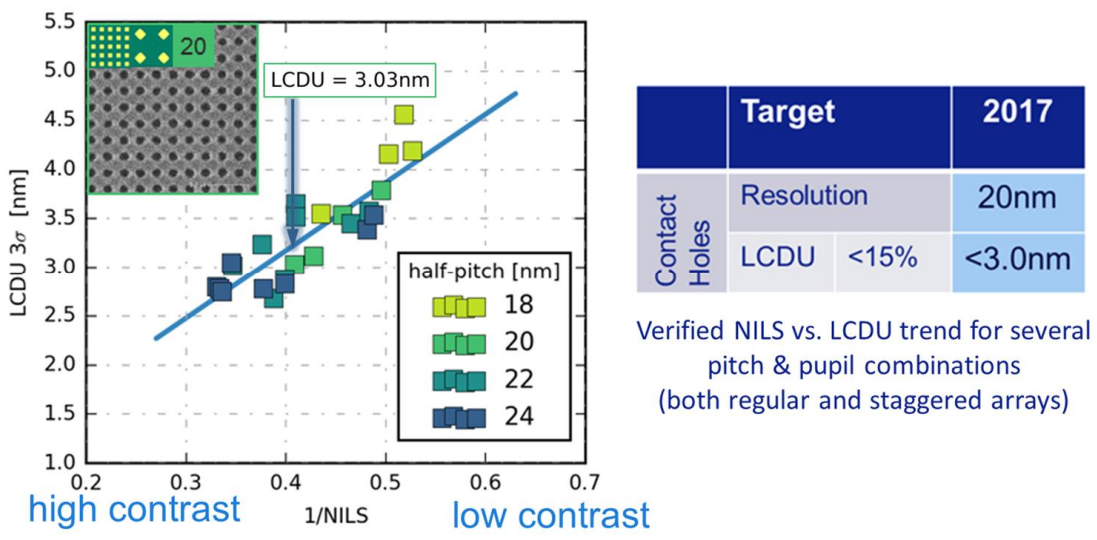

Figure 6. NXE:3400 LCDU results dense contact holes meet $5 \mathrm{~nm}$ node requirements.

Figure 6 shows more data points for LCDU of CHs for several pitch and pupil combinations and target for CH for 2017. Higher Scanner contrast enables lower LCDU. The highlighted data point meets the 5 nm node requirement on resolution and LCDU. Further improving LCDU by post processing after lithography can be very beneficial. Figure 7 shows that etching can improve the LCDU up to $40 \%$ in CAR resists ${ }^{20}$. 


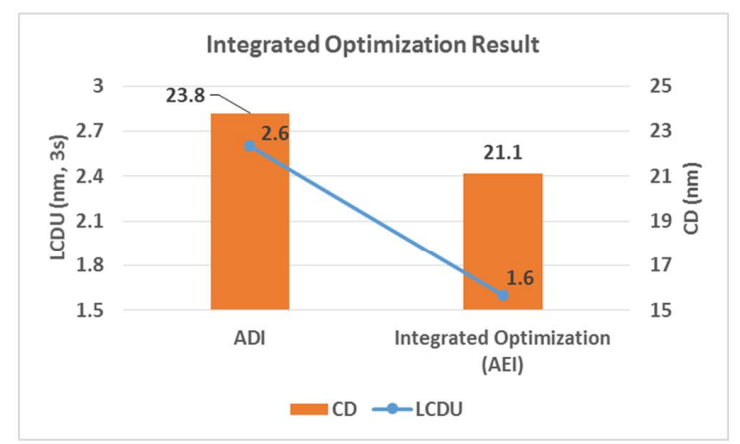

Target Oxide Level

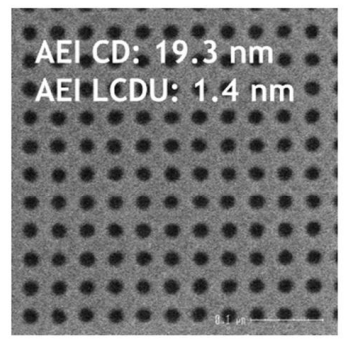

Resist Level

Figure 7. LCDU reduction with etch for CAR resist ${ }^{20}$.

\subsection{Process Optimization to tune Sensitivity (Dose) and Roughness (LWR)}

In Figure 8, $16 \mathrm{~nm}$ Dense Line patterns of Inpria YA Series resist patterns are shown. All data points and images correspond to a different PEB temperature. By increasing PEB temperature from $90^{\circ} \mathrm{C}$ to $150^{\circ} \mathrm{C}$, the dose-to-size value which is also named best energy (BE), is decreased from 47.5 to $24 \mathrm{~mJ} / \mathrm{cm}^{2}$. The decrease in the dose value resulted in increased roughness (LWR) as expected. These results clearly show the trade-off relation between Dose and LWR for this resist platform. The optimum dose-LWR combination depends on the application. For further improvement, a certain PEB temperature can be decided and additional optimizations such as varying resist film thickness, under-layer, hard bake etc. can be applied.

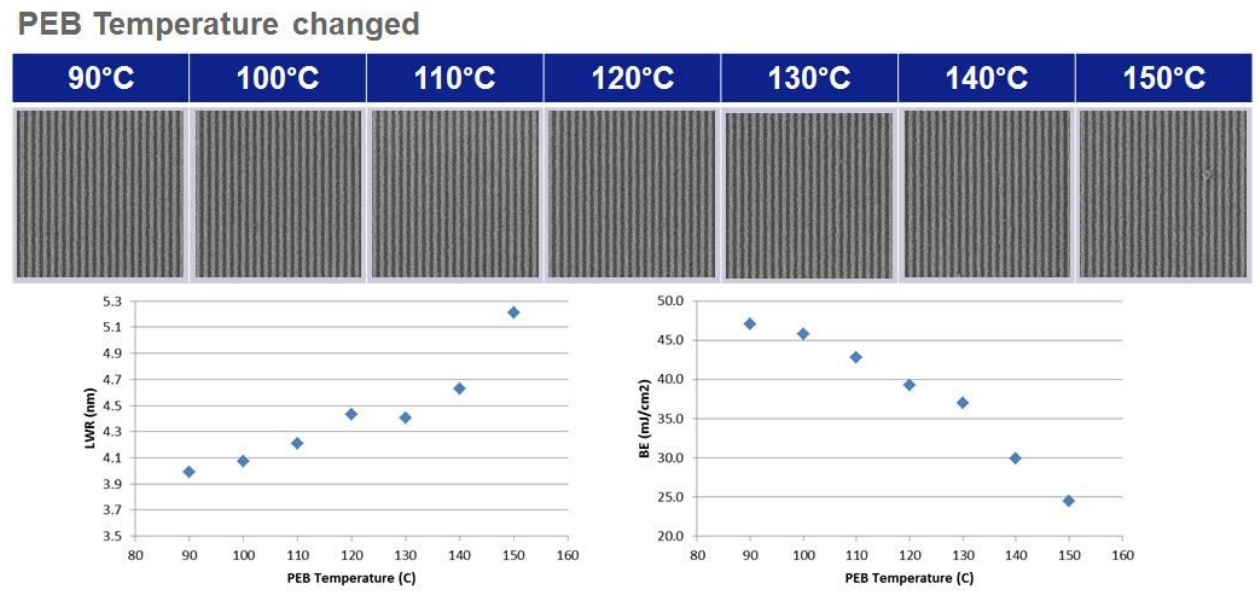

Figure 8: Process optimization on $16 \mathrm{~nm}$ hp dense line patterns of Inpria YA Series resist using different post exposure bake (PEB) temperatures.

PEB is a knob among others and there is a large room in process optimization to get the desired outcome. By only changing the PEB temperature, a considerable effect on the dose is obtained. According to Hinsberg et.al. generation of dissolution contrast in Inpria metal-oxide photoresists is driven by differential condensation of oxo-networks. Photoproducts are generated on exposure form 3D networks through a condensation process ${ }^{21}$. PEB drives this oxonetwork formation with the effect of more complete network formation with increased PEB temperature. This leads to less exposure dose required to drive condensation in the resist. In that sense baking and exposing the resist have a similar effect on the resist. Upon exposure, the condensation level, material composition and density of the resist is expected to change with respect to the unexposed part. It is important to keep in mind that, unlike exposure, baking affects the whole resist, thus a contrast loss may be expected at elevated temperatures. Results show that the patterns are preserved even with high baking temperatures up to $150^{\circ} \mathrm{C}$. 
Like the dose, roughness is also tuned by PEB temperature. With lower PEB, a higher dose is needed which results in lower roughness, due to some combination of reduced noise in the oxo-network formation and better photon statistics. It would be interesting to see what other knobs can be tuned to affect dose and LWR. Resist composition (i.e. metal-oxide cluster size), resist profile control (i.e. by resist thickness, absorptivity, under-layer or rinse material), development behavior (i.e. by stronger developers) can be some directions to look into.

\subsection{High Resolution Performance and Process Window of EUV Resists:}

Figures 9 and 10 show the process windows of CAR and non-CAR EUV resists in detail, with multiple images through focus and dose. These results are from focus exposure matrix (FEM) exposures, where dose and focus values are changed step by step. The yellow dotted lines show the dose range through focus where the CD value is within $\pm 10 \%$ of the target $\mathrm{CD}$. The blue solid line marks the region where the line quality is $\mathrm{OK}$ and at the same time $\mathrm{CD}$ value is within $10 \%$ of target hp, which is also named as overlapping process window. The SEM images of resist patterns with different focus values and same dose are shown below the graphs. The SEM images with the same focus value and different dose values are shown to the right of the graph. The lines connect the images to the Dose and Focus values in the graph. The green line around the SEM image indicates the pattern quality where the SEM image is taken is good. The OK/NOK decision is made by using CD images, and the LWR images are used in the figure. As seen the $13 \mathrm{~nm}$ results are not from a single data point but outcome of lines printed through a large dose and focus region.

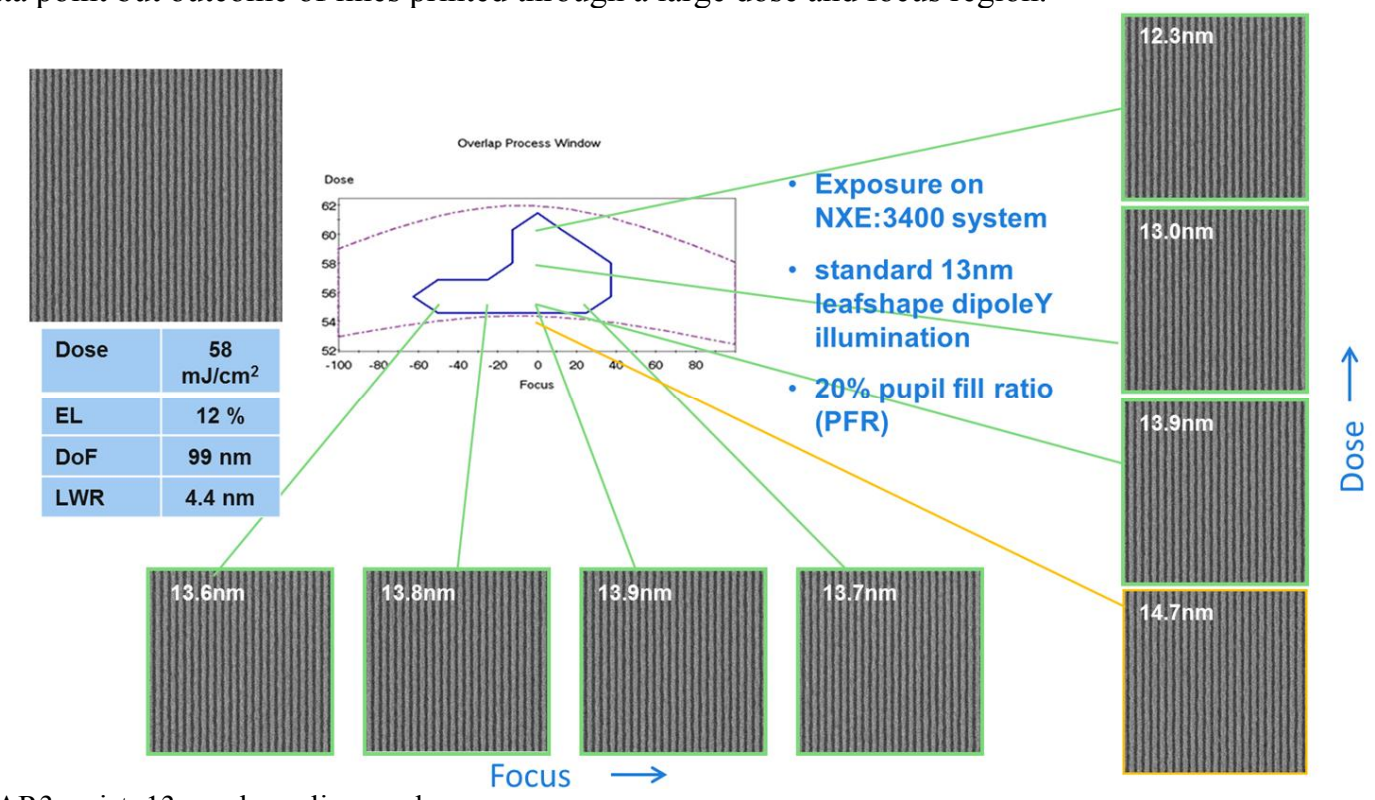

Figure 9. CAR3 resist: $13 \mathrm{~nm}$ dense lines and spaces.

CARs are the current industry standard for DUV lithography and have been utilized for EUV lithography for many years. With the increased demand for high resolution, resist suppliers have been modifying EUV CAR resists to meet the challenging requirements. Figure 9 shows the new CAR resist process window. The resist is exposed at a wide dose and focus range. Exposure was done on NXE:3400 system, using standard 13nm leaf-shape dipoleY illumination with 20\% PFR. This pupil can be made without light loss, thus no throughput loss. Over the last year, EUV CAR resist have shown progress in the sense that CAR now has $13 \mathrm{~nm}$ process window unlike previous year ${ }^{1}$. 


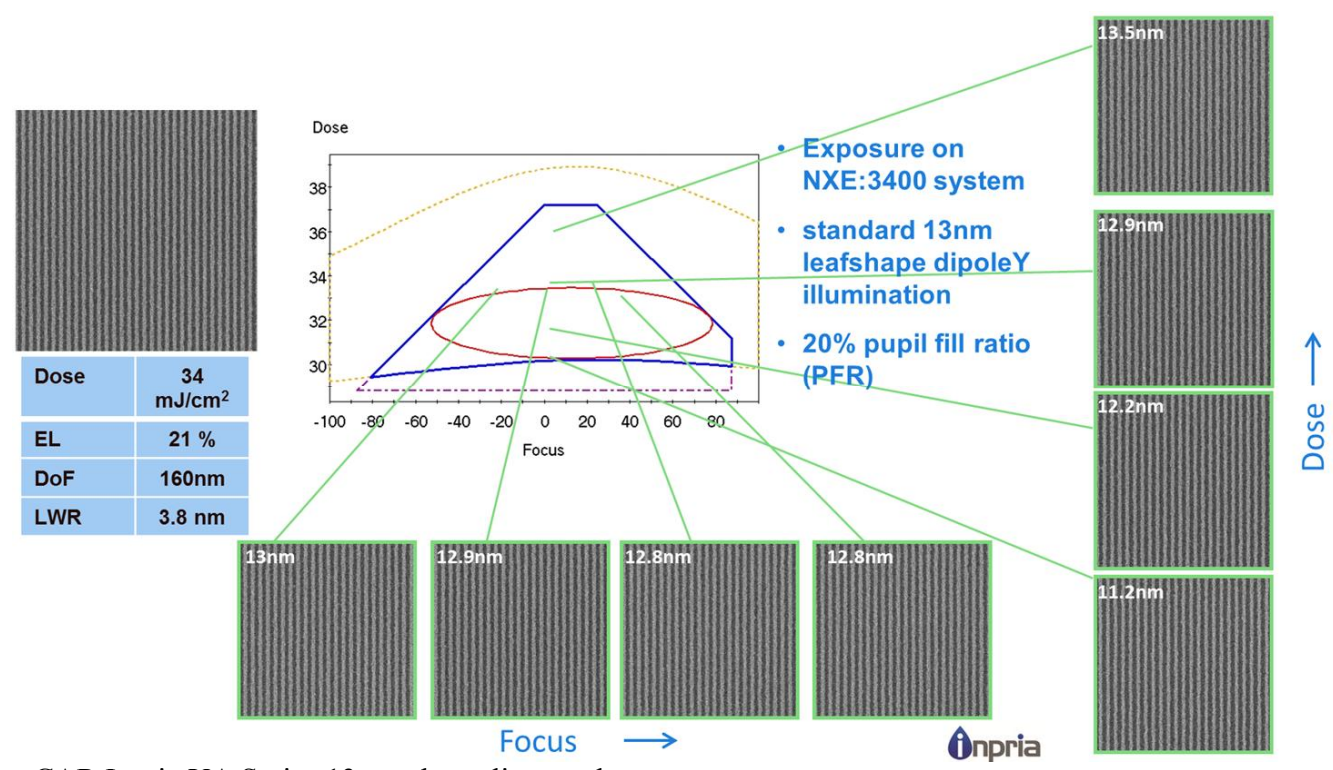

Figure 10. Non-CAR Inpria YA Series:13 nm dense lines and spaces.

Figure 10 shows $13 \mathrm{~nm}$ dense lines and spaces of non-CAR Inpria YA Series resist, over a wide dose and focus range. The process window is larger compared to CAR resist, with relatively lower LWR and dose. The Inpria YA Series resist has low dose sensitivity which enables high exposure latitude values and contributes to large process window. This high $13 \mathrm{~nm}$ performance with large process window is routinely verified by multiple wafers. The exposure conditions are same with the conditions used for CAR resist.

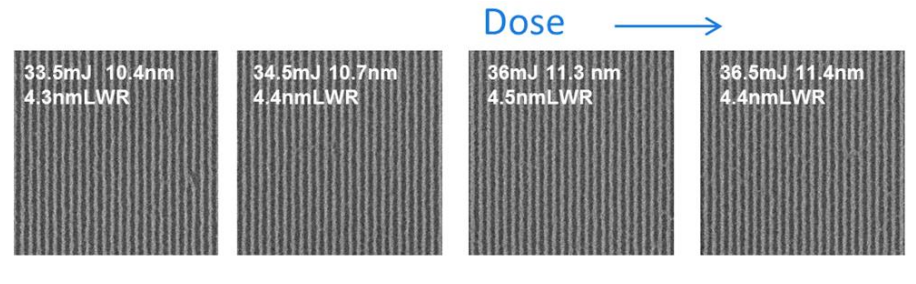

Improvement needed with process optimization
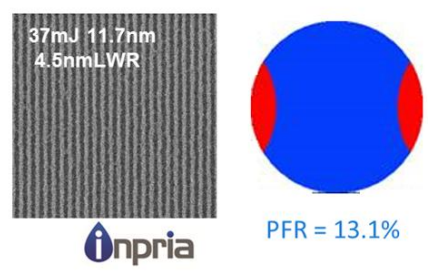

\begin{tabular}{|c|c|}
$\begin{array}{c}\text { Best } \\
\text { Energy }\end{array}$ & $\begin{array}{c}\mathbf{3 8} \\
\mathbf{m J} / \mathrm{cm}^{2}\end{array}$ \\
\hline LWR & $4.7 \mathrm{~nm}$ \\
\hline
\end{tabular}

- Exposure on NXE:3400 system

- 12nm leafshape dipole $X$

- $13.1 \%$ pupil fill ratio (PFR)

Figure 11. Inpria YA-Series Resist $12 \mathrm{~nm} \mathrm{~V} \mathrm{dense} \mathrm{lines} \mathrm{through} \mathrm{dose} 12 \mathrm{~nm}$ resolution is shown with Inpria YA Series EUV resist on NXE: 3400 scanner.

As shown in Figure 11, 12nm dense lines are resolved through dose with Inpria YA-Series non-CAR resist. NXE: 3400 scanner is qualified for a resolution of $13 \mathrm{~nm}$ line spaces ${ }^{19}$. To explore the limits of the imaging capability we exposed, $12 \mathrm{~nm}$ lines and spaces using a leaf-shape DipoleX illumination pupil with pupil fill ratio of $13.1 \%$ on the NXE: 3400 . This pupil, designed to give sufficient contrast for $12 \mathrm{~nm}$ gave $\sim 40 \%$ transmission loss compared to standard $13 \mathrm{~nm}$ pupil (PFR 20\%). To cover a large dose range, for $12 \mathrm{~nm}$ lines and spaces, a meander exposure was chosen at "0" nominal focus (so there is no multiple focus values and DOF assessment). There are several defects such as bridging. The line quality is poor compared to $13 \mathrm{~nm}$ patterns and improvement needed with process optimization. 


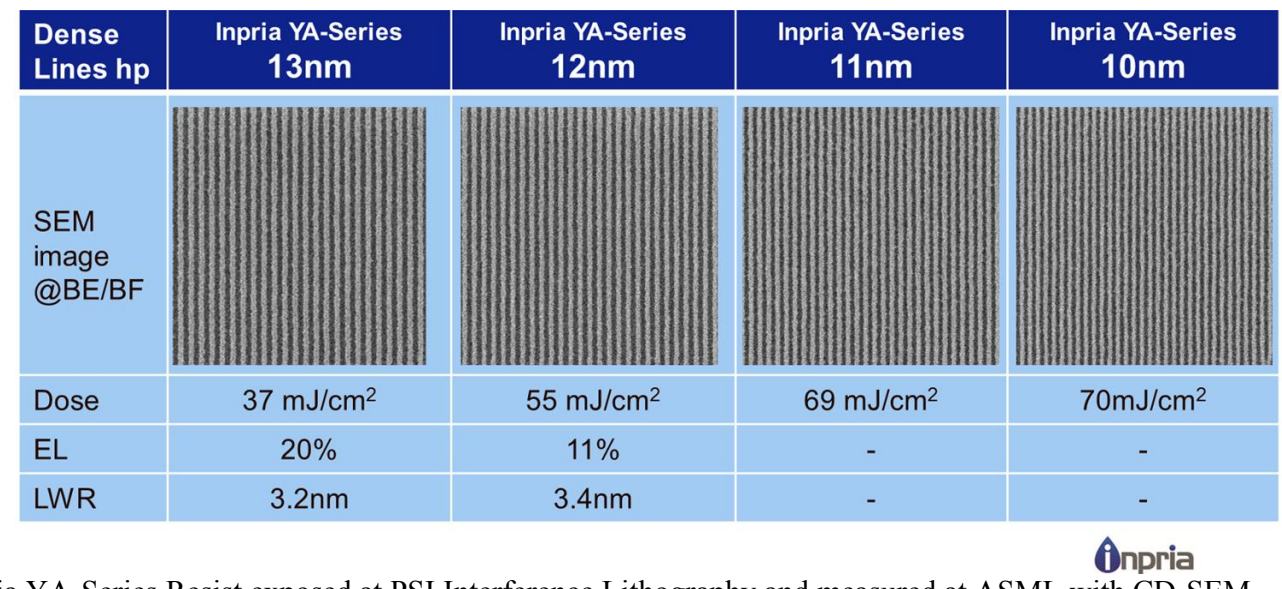

Figure 12. Inpria YA-Series Resist exposed at PSI Interference Lithography and measured at ASML with CD-SEM.

13 and $12 \mathrm{~nm}$ performance of EUV resists on NXE: 3400 EUV scanner was discussed above. In order to evaluate the extendibility of these resist for smaller features, we investigated their performance down to $10 \mathrm{~nm}$ resolution using EUVIL tool at PSI (Fig. 12). The exposed Inpria YA-Series sample was measured at ASML. All 13, 12, 11 and 10nm can be printed through dose, while only $13 \mathrm{~nm}$ and $12 \mathrm{~nm}$ showed good line quality and exposure latitude (Fig. 12). With a mask having features smaller than $10 \mathrm{~nm}$, and improved mask quality, beyond $10 \mathrm{~nm}$ performance can be expected. There is a trend in the increased dose while going to smaller hp values. This trend was not observed in ASML exposures where $13 \mathrm{~nm}$ and $12 \mathrm{~nm}$ gave similar dose values. PSI interference lithography tool and ASML scanner tool have very different working principles, thus different ways of image formation which could explain the two opposite trends ${ }^{16,17}$. We note that the reported dose values are tentative estimations and further experiments are needed for the exact dose to size values.

\subsection{Next Steps towards EUV lithography to enable HVM}

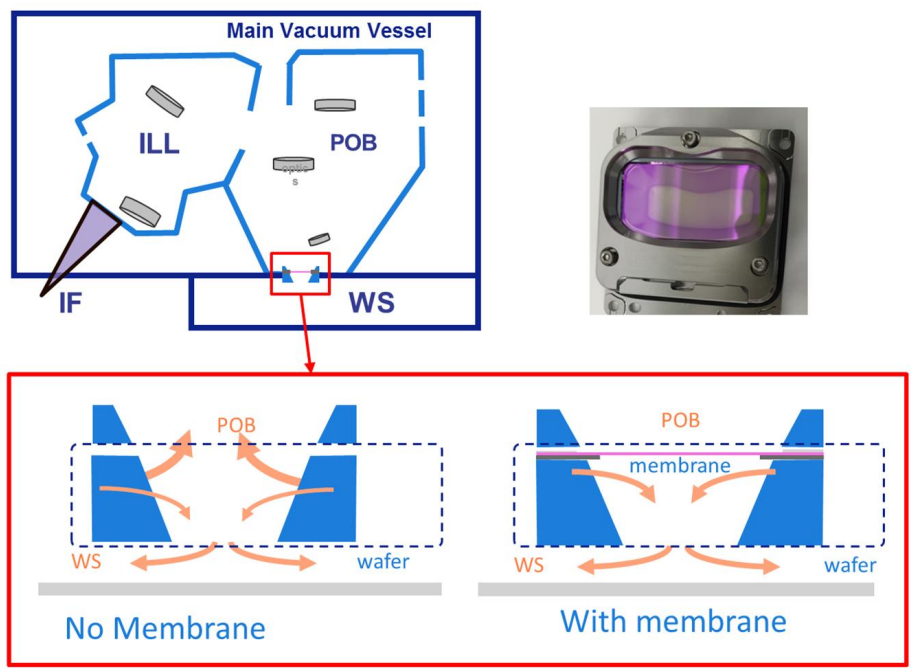

Figure 13. DGL membrane acts as a physical outgassing barrier to protect POB.

From alpha-demo tool till today, dynamic gas lock has been used in the NXE scanners to separate the projection optics box (POB) from the wafer stage (WS) compartment. The downward flow has been highly effective in suppressing the hydrocarbon species which may outgas from the resist during exposure. This suppression may not be as efficient for heavy metal hydrides. Currently a dynamic gas lock (DGL) membrane is introduced which forms a physical barrier between POB and WS (Fig. 13). This will enable 100\% suppression of outgassing species and complete protection of 
POB. Introducing a membrane between wafer and optics allows a much wider range of resist materials to be used. The details on the membrane and its uses other than outgassing suppression are discussed by Kerkhof et al. ${ }^{19}$.

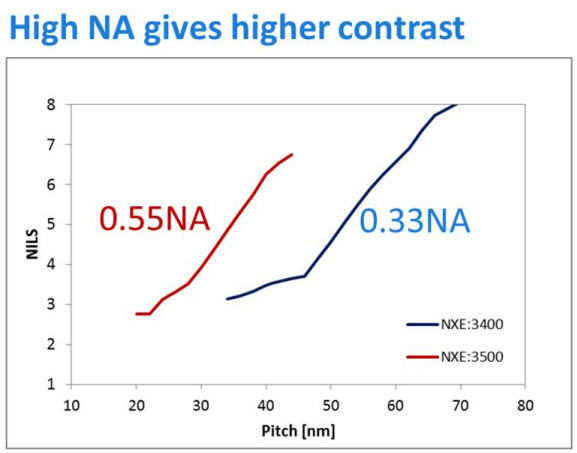

High NA gives lower LCDU

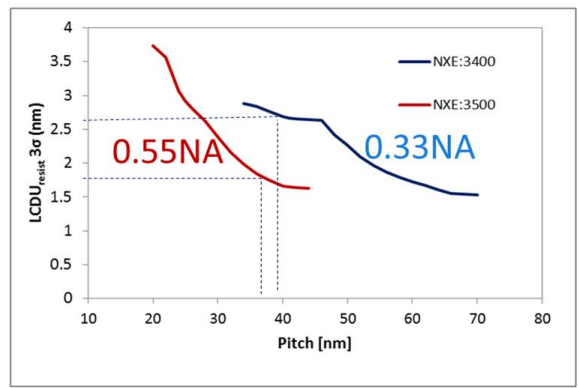

Validated model for current CAR resist shows $3 \mathrm{~nm}$ node is possible with $0.55 \mathrm{NA}$

\begin{tabular}{|c|c|c|c|}
\hline & Target & 2017 & 2020 \\
\hline \multirow{2}{*}{ 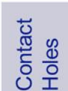 } & Resolution & $20 \mathrm{~nm}$ & $18 \mathrm{~nm}$ \\
\hline & LCDU & $<3.0 \mathrm{~nm}$ & $<2.7 \mathrm{~nm}$ \\
\hline
\end{tabular}

Figure 14. High-NA will give significant contrast and LCDU improvement (regular CHs).

For further shrink a high-NA NXE system has been introduced ${ }^{2}$. Figure 14-left, shows the expected contrast improvement calculated by simulations. For a given pitch $0.55 \mathrm{NA}$ has much higher contrast compared to $0.33 \mathrm{NA}$. With the improved contrast, higher resolution, lower dose, lower LWR and LCDU is expected. The validated LCDU model discussed earlier is used to show expected LCDU for given pitch with current and high-NA cases (Fig. 14, right). The validated LCDU model using the CAR2 resist shows $3 \mathrm{~nm}$ node is possible with $0.55 \mathrm{NA}$.

\section{CONCLUSION}

In this paper we discussed improvements in resist performance towards EUV high volume manufacturing. EUV resists have made significant progress on resolution over last decade. We report 13 and $12 \mathrm{~nm} \mathrm{hp} \mathrm{resolution} \mathrm{on} \mathrm{NXE} \mathrm{scanners}$ and down to $10 \mathrm{~nm}$ resolution on PSI interference lithography tool, limited by the employed mask resolution. We introduce a LCDU model having experimentally determined resist input parameters and show its validation with the experimental CH data. LCDU has been improved through resist chemistry, post-litho processing as well as improved scanner aerial image contrast. With the state-of-the-art imaging we show $\mathrm{CH}$ data which meets $5 \mathrm{~nm}$ node requirements. Membrane introduced between wafer and optics acts as outgassing barrier and enables use of wider class of resist materials. We believe this will support development of higher performing resists. Finally, our validated LCDU model indicates that improved aerial image with high-NA scanner can meet $3 \mathrm{~nm}$ node LCDU requirements.

\section{Acknowledgement}

We would like to thank to the material suppliers for the various materials used, to Peter de Loijer, Dorothe Oorschot and Yin Fong Choi for metrology support, Gazi Tanriseven, Karen Garrido Olvera, Mike van Gils and Rolf Custers for exposure support, to Joep van Dijk for arranging exposures at ASML.

\section{REFERENCES}

[1] Pirati, A., "EUV lithograpy performance for manufacturing: status and outlook," Proc. SPIE 97760A, 1-15 (2016).

[2] Van Schoot, J.,"EUV High-NA scanner and mask optimization for sub 8 nm resolution," Proc. SPIE 97761I, 115 (2016). 
[3] De Simone, D., "Demonstration of an N7 integrated fab process for metal oxide EUV photoresist," Proc. SPIE 97760B, 1-9 (2016).

[4] Wallow, T., "Evaluation of EUV resist materials for use at the $32 \mathrm{~nm}$ half-pitch node," Proc. SPIE 69211F, 1-11 (2008).

[5] Thackeray, J. W., "Materials challenges for sub-20nm lithography," J. Micro/Nanolith. MEMS MOEMS 10(3) 033009, 1-8 (2011).

[6] Gallatin, G. M., "Resist blur and line edge roughness," Proc. SPIE 5754, 38-52 (2005).

[7] Itani, T., Kozawa, T., "Resist Materials and Processes for Extreme Ultraviolet Lithography ," Jpn. J. Appl. Phys 52 010002, 1-14 (2013).

[8] Kozawa, T., Tagawa, S., "Radiation Chemistry in Chemically Amplified Resists," Jpn. J. Appl. Phys 49 030001, 1-19 (2010).

[9] Mack, C. A., Thackeray, J. W., Biafore, J. J., Smith, M. D., "Stochastic exposure kinetics of extreme ultraviolet photoresists: simulation study," J. Micro/Nanolith. MEMS MOEMS 10(3) 033019, 1-11 (2011).

[10] Kozawa, T., Saeki, A., Tagawa, S., "Modeling and simulation of chemically amplified electron beam, x-ray, and EUV resist processes," J. Vac. Sci. Technol. B 22(6), 3489-3492 (2004).

[11] Gronheid, R., "EUV secondary electron blur at the 22nm half pitch node," Proc. SPIE 796904, 1-11 (2011).

[12] Gronheid, R., "EUV Resist Requirements: Absorbance and Acid Yield," Proc. SPIE 727332, 1-8 (2009).

[13] Grenville, A., "Integrated Fab Process for Metal Oxide EUV Photoresist," Proc. SPIE 94250S, 1-8 (2015).

[14] Stowers, J., "Metal Oxide EUV Photoresist Performance for N7 Relevant Patterns and Processes," Proc. SPIE 977904, 1-11 (2016).

[15] Buitrago, E., Fallica, R., Fan, D., Kulmala, T. S., Vockenhuber, M., Ekinci, Y., "SnOx high-efficiency EUV interference lithography gratings towards the ultimate resolution in photolithography," Microelectronic Engineering 155, 44-49 (2016).

[16] Buitrago, E., Nagahara, S., Yildirim, O., Nakagawa, H., Tagawa, S., Meeuwissen, M., Nagai, T., Naruoka, T., Verspaget, C., Hoefnagels, R., Rispens, G., Shiraishi, G., Terashita, Y., Minekawa, Y.,Yoshihara, K., Oshima, A., Vockenhuber, M., Ekinci, Y., "Sensitivity enhancement of chemically amplified resists and performance study using extreme ultraviolet interference lithography," J. Micro/Nanolith. MEMS MOEMS 15(3), 033502, 1-9 (2016).

[17] Ekinci, Y., "EUV resists towards 11 nm half-pitch,” Proc. SPIE 904804, 1-10 (2014).

[18] Finders, J., "Contrast optimization for 0.33NA EUV Lithography," Proc. SPIE 97761P, 1-9 (2016).

[19] Van de Kerkhof, M., "Enabling sub-10nm node lithography: presenting the NXE:3400B EUV," Proc. SPIE, in publication, (2017).

[20] Liang, A., "Integrated Approach to Improving Local CD Uniformity in EUV Patterning," Proc. SPIE, in publication, (2017).

[21] Hinsberg, W. D., "A numeric model for the imaging mechanism of metal oxide EUV resists," Proc. SPIE, SPIE, in publication, (2017). 